\title{
Impacto dos transtornos depressivos e ansiosos sobre as manifestações da menopausa
}

\author{
Impact of depressive and anxiety disorders over menopause manifestations
}

\author{
Andre Barciela Veras ${ }^{1}$, Arabella Rassi ${ }^{2}$, Livia Mitsue Gomes Yukizaki ${ }^{3}$, Luisa Duarte Novo ${ }^{3}$, \\ Flávia Schueler Franco ${ }^{3}$, Antonio Egídio $\mathrm{Nardi}^{4}$
}

${ }^{1}$ Mestre. Pesquisador. ${ }^{2}$ Residência Médica em Psiquiatria. Pesquisadora. ${ }^{3}$ Estudante de Medicina. Aluna de Iniciação Científica. ${ }^{4}$ Livre-docente. Professora associada, Faculdade de Medicina, Universidade Federal do Rio de Janeiro (UFRJ), Rio de Janeiro, RJ.

Este trabalho foi realizado no Instituto de Psiquiatria, Universidade Federal do Rio de Janeiro (UFRJ), Rio de Janeiro, RJ

Estudo financiado pelo CNPq (402846/2005-2).

\section{Resumo}

Objetivo: Determinar, pela primeira vez, em uma amostra ambulatorial brasileira, o impacto dos diagnósticos psiquiátricos sobre os sintomas da menopausa presentes no índice de menopausa de Blatt-Kupperman (B-K).

Métodos: Avaliamos consecutivamente, através do instrumento diagnóstico estruturado MINI 4.4 e da entrevista psiquiátrica tradicional, mulheres $(\mathrm{n}=86)$ em atendimento no ambulatório de menopausa do Instituto de Ginecologia da Universidade Federal do Rio de Janeiro. As pacientes incluídas no estudo $(n=48)$ foram avaliadas pela escala B-K até 6 meses antes ou depois da entrevista pelo MINI e divididas em dois grupos: o grupo com algum transtorno depressivo-ansioso (GTDA) $(\mathrm{n}=26)$ e o grupo-controle $(\mathrm{GC})$ $(\mathrm{n}=22)$.

Resultados: O GTDA apresentou uma pontuação significativamente maior do que o GC na escala de B-K (22,6 versus 13,7). Entre os sintomas significativamente mais intensos no GTDA estão parestesia e melancolia. Além disso, observamos que as pacientes do GTDA queixavam-se de um maior número de sintomas do que as pacientes do GC (6,8 vs. 4,8). Entre os sintomas significativamente mais relatados no GTDA, estão parestesia, fraqueza e melancolia.

Conclusão: Apesar da limitação da pequena amostra, pudemos observar o significativo viés dos transtornos depressivos e ansiosos sobre as pacientes em tratamento por queixas referentes à menopausa. Por esse motivo, o perfil sintomático de cada paciente deve ser sempre observado, evitando o ginecologista basear-se na intensidade global dos sintomas do B-K para decidir sobre o tratamento. Descritores: Depressão, ansiedade, menopausa, índice de Blatt-Kupperman.

\begin{abstract}
Objective: To establish for the first time in a Brazilian outpatient sample the impact of depressive and anxiety disorders over the symptoms of the Blatt-Kupperman menopausal index (B-K).

Methods: Women $(n=86)$ receiving care in the menopause clinic at Instituto de Ginecologia da Universidade Federal do Rio de Janeiro were consecutively assessed using a structured diagnostic instrument (MINI 4.4) and a traditional psychiatric interview. Patients included in the study $(n=48)$ were assessed using the B-K scale within 6 months before or after the MINI. The total sample was divided into the anxiety and depressive disorder group (GTDA) $(n=26)$ and the control group $(C G)(n=22)$.

Results: B-K total scores were greater in the GTDA comparing with the CG (22.6 vs. 13.7). Paresthesia and melancholia were significantly more severe in the GTDA. GTDA patients also complained of a greater number of symptoms (6.8 vs. 4.8$)$ than the GC. Paresthesia, weakness and melancholia were significantly more present in the GTDA.

Conclusion: Although the small sample limitation we could observe a great bias of depressive and anxiety disorders over menopause outpatients. For that reason, the symptomatic profile of each patient must always be observed by the gynecologist, avoiding exclusive observation of B-K total score to decide about the treatment.
\end{abstract}

Keywords: Depression, anxiety, menopause, Blatt-Kupperman index. 


\section{Introdução}

A menopausa é conhecida como um período de alterações globais do corpo e da mente da mulher. Entre os sintomas psicológicos, o humor é uma das estruturas mais acometidas. Durante esse período, mulheres que tiveram um transtorno depressivoansioso ao longo da vida têm uma maior probabilidade de apresentarem outro episódio ${ }^{1}$, enquanto outras podem sentir pela primeira vez insônia, irritabilidade, alterações de humor, ausência de desejo sexual e fadiga ${ }^{2}$. Alguns desses sintomas fazem parte do índice de menopausa de BlattKupperman (B-K) ${ }^{3}$, um dos instrumentos mais usados na prática clínica para quantificar os sintomas da menopausa e avaliar a evolução ao longo do tempo ${ }^{4}$.

Além de serem queixas comuns na menopausa, os sintomas emocionais são as queixas mais freqüentes entre as mulheres brasileiras que procuram atendimento ginecológico. Em um levantamento domiciliar brasileiro que contou com 367 mulheres, Pedro et al. ${ }^{5}$ observaram que a intensidade dos sintomas psicológicos é positivamente relacionada com a procura por tratamento. Whitehead et al. ${ }^{6}$ também observaram que a gravidade da ansiedade, irritabilidade e “depressão" era o principal diferencial entre mulheres que procuram ou não algum tratamento. Esse viés na procura por tratamento pode levar a uma concentração de mulheres acometidas por um transtorno depressivo ou ansioso nos ambulatórios de ginecologia e menopausa.

Após observarmos a alta incidência de transtornos depressivo-ansiosos no ambulatório de menopausa do Instituto de Ginecologia da Universidade Federal do Rio de Janeiro em estudo prévio $^{7}$, procuramos determinar, pela primeira vez em uma amostra ambulatorial brasileira, a provável forte influência dos diagnósticos psiquiátricos sobre os sintomas da menopausa presentes no B-K.

\section{Métodos}

Avaliamos consecutivamente, através do instrumento diagnóstico estruturado MINI $4.4^{8}$ e da entrevista psiquiátrica, mulheres $(n=86)$ em atendimento no ambulatório de menopausa do Instituto de Ginecologia da Universidade Federal do Rio de Janeiro, objetivando a determinação de dados publicados em estudo prévio ${ }^{7}$. Foram coletadas, ainda, variáveis demográficas e da história ginecológica e psiquiátrica. Todas as avaliações foram realizadas pelo mesmo psiquiatra. Posteriormente, a escala de B-K foi obtida através do prontuário das pacientes. Todas as escalas foram aplicadas pela mesma ginecologista. As pacientes incluídas no estudo $(\mathrm{n}=48)$ foram aquelas avaliadas pela escala B-K até 6 meses antes ou depois da entrevista pelo MINI. Foram excluídas as pacientes cuja avaliação ginecológica dos sintomas da menopausa havia sido realizada mais de 6 meses antes ou depois da avaliação psiquiátrica. Avaliamos que uma diferença temporal entre as avaliações maior do que esse período poderia comprometer as associações entre os dados dos instrumentos utilizados.

Dividimos as pacientes em dois grupos. O grupo com algum transtorno depressivo-ansioso (GTDA) - composto por 26 mulheres - e o grupo-controle (GC) - composto por 22 mulheres - sem qualquer diagnóstico psiquiátrico pelo MINI e sem história de qualquer tratamento psiquiátrico no momento da avaliação.

O B-K é constituído de 11 sintomas, que são categorizados por uma escala de 4 pontos, onde $0=$ ausente e 3 = grave. Alguns sintomas têm um valor maior: fogacho tem um peso de 4, e nervosismo, insônia e parestesia têm peso 2. A pontuação total é categorizada como leve (15 a 20), moderada (21 a 35) e grave $(>35)^{3}$.

O Instituto de Ginecologia da Universidade Federal do Rio de Janeiro fica situado em uma posição central da cidade do Rio de Janeiro. Caracteriza-se por ser um centro de baixa complexidade e visa prestar atendimento à população da vizinhança. Por ser um hospital de atendimento medico público, a maioria das pacientes tem um baixo nível econômico e cultural.

Todas as pacientes foram informadas sobre os objetivos do estudo e concordaram em participar da entrevista. O protocolo foi aprovado pelo comitê de ética local, e as pacientes assinaram o termo de consentimento livre e esclarecido.

A média e o desvio padrão (DP) foram utilizados para avaliar os dados descritivos das variáveis de distribuição normal e mediana e percentuais 25 e 75\% para as variáveis de distribuição não-normal. O teste exato de Fisher e o $\chi^{2}$ foram utilizados para comparar as variáveis categóricas, e o teste $t$ de student e o de Mann-Whitney foram utilizados para comparar as variáveis contínuas entre os grupos. Um valor de $\mathrm{p}$ $<0,1$ e $>0,05$ foi considerado como tendência, e p = 0,05 foi considerado como estatisticamente significativo.

\section{Resultados}

Entre as mulheres incluídas no estudo ( $\mathrm{n}=48)$, observamos $33,3 \%$ de transtornos ansiosos e $18,7 \%$ 
Tabela 1 - Comparação dos dados demográficos entre o grupo com transtorno depressivo ou ansioso e o grupo-controle

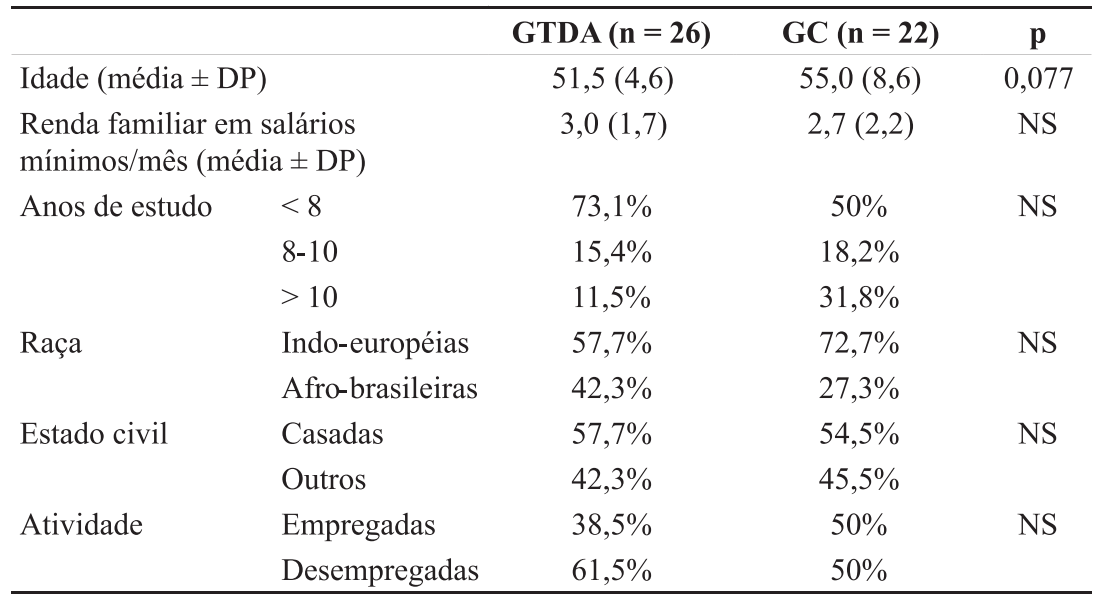

Tabela 2 - Comparação das variáveis ginecológicas e clínicas entre os grupos

\begin{tabular}{lccc}
\hline Parâmetros clínicos & GTDA (26) & GC (22) & p \\
\hline Menarca - mediana (25-75\%) & $12(11,0-13,7)$ & $13(11,8-14,2)$ & NS \\
Terapia de reposição hormonal & $26,9 \%$ & $31,8 \%$ & NS \\
Tipo de menopausa & & & \\
$\quad$ Natural & $76,9 \%$ & $72,7 \%$ & NS \\
$\quad$ Cirúrgica & $23,0 \%$ & $27,2 \%$ & \\
Estágio da menopausa & & & \\
$\quad$ Pré-menopausa & $3,8 \%$ & $0 \%$ & NS \\
$\quad$ Perimenopausa & $15,3 \%$ & $9,0 \%$ & \\
$\quad$ Pós-menopausa & $80,7 \%$ & $90,9 \%$ & \\
História pessoal de depressão & $26,9 \%$ & $31,8 \%$ & NS \\
História familiar de depressão & $46,1 \%$ & $18,1 \%$ & 0,065 \\
\hline
\end{tabular}

de transtornos depressivos como diagnósticos principais. No GTDA $(n=26)$, mais da metade tinha pelo menos uma comorbidade (53,8\%).

As comparações dos dados demográficos, ginecológicos e psiquiátricos entre o GTDA e o GC podem ser observadas nas Tabelas 1 e 2 . A diferença desses dados entre os grupos não se mostrou estatisticamente significativa.

O GTDA apresentou uma pontuação significativamente maior do que o GC na escala de B-K (22,6 versus 13,7). Entre os sintomas significativamente mais intensos no GTDA, estão parestesia e melancolia. Além disso, observamos que as pacientes do GTDA queixavam-se de um maior número de sintomas do que as pacientes do GC $(6,8$ versus 4,8). Entre os sintomas significativamente mais relatados no GTDA, estão parestesia, fraqueza e melancolia. Apresentamos, nas Tabelas 3 e 4, as comparações de cada sintoma entre os grupos.

\section{Discussão}

Observamos que, além de queixarem-se de forma mais intensa dos sintomas da menopausa, pacientes com transtornos depressivos ou ansiosos queixamse de um maior número de sintomas físicos e psíquicos. Observação semelhante foi descrita por inúmeros estudos que abordam a relação entre ansiedade e depressão e somatização em serviços 
Tabela 3 - Comparação da intensidade dos sintomas da menopausa (índice de menopausa de Blatt-Kupperman) entre os grupos

\begin{tabular}{lccc}
\hline Sintomas & $\begin{array}{c}\text { GTDA mediana } \\
(\mathbf{2 5 - 7 5 \% )}\end{array}$ & $\begin{array}{c}\text { GC mediana } \\
\mathbf{( 2 5 - 7 5 \% )}\end{array}$ & p \\
\hline Fogachos & $8(0-12)$ & $0(0-8)$ & 0,095 \\
Nervosismo & $2(0-6)$ & $0(0-4)$ & $\mathrm{NS}$ \\
Insônia & $4(0-6)$ & $0(0-4)$ & 0,057 \\
Parestesia & $2(2-4)$ & $0(0-2)$ & 0,007 \\
Melancolia & $1(0-2)$ & $0(0-0)$ & 0,002 \\
Cefaléia & $0(0-1)$ & $1(0-1)$ & $\mathrm{NS}$ \\
Artralgia ou mialgia & $2(1-3)$ & $1(0-3)$ & $\mathrm{NS}$ \\
Fraqueza & $1,5(1-3)$ & $0(0-3)$ & 0,075 \\
Vertigem & $1(0-1)$ & $0(0-1)$ & $\mathrm{NS}$ \\
Formigamento & $0(0-0)$ & $0(0-1)$ & $\mathrm{NS}$ \\
Palpitação & $1(0-2)$ & $1(0-1)$ & $\mathrm{NS}$ \\
Total - média (DP) & $22,6(9,9)$ & $13,7(9,4)$ & 0,003 \\
\hline
\end{tabular}

$\mathrm{DP}=$ desvio padrão; GC = grupo-controle; GTDA = grupo com algum transtorno depressivo-ansioso; NS = não-significante.

Tabela 4 - Comparação entre os grupos da quantidade de mulheres que se queixam de cada sintoma da escala de Blatt-Kupperman

\begin{tabular}{lccc}
\hline Sintomas & GTDA (\%) & GC (\%) & p \\
\hline Artralgia ou mialgia & 80,7 & 59,0 & NS \\
Parestesia & 76,9 & 40,9 & 0,018 \\
Fraqueza & 76,9 & 40,9 & 0,018 \\
Fogachos & 73,0 & 45,4 & 0,077 \\
Insônia & 73,0 & 45,4 & 0,077 \\
Nervosismo & 65,3 & 45,4 & NS \\
Melancolia & 61,5 & 9,0 & $<0,001$ \\
Vertigem & 61,5 & 36,3 & NS \\
Palpitação & 61,5 & 59,0 & NS \\
Cefaléia & 38,4 & 59,0 & NS \\
Formigamento & 19,2 & 40,9 & NS \\
Número de sintomas - média (DP) & $6,8(2,4)$ & $4,8(2,6)$ & 0,007 \\
\hline
\end{tabular}

$\mathrm{DP}=$ desvio padrão; GC = grupo-controle; GTDA = grupo com algum transtorno depressivo-ansioso; NS = não-significante.

de atendimento primário. Waal et al. ${ }^{9}$, em estudo transversal com 1.458 pacientes, observaram uma razão de chance maior que seis para a presença de sintomas como falta de energia, fadiga e esquecimento entre pacientes com mais sintomas depressivo-ansiosos. Em um estudo recente, conduzido em ambiente ambulatorial para cuidados referentes à menopausa, Callegari et al. ${ }^{10}$ observaram, entre as pacientes deprimidas, níveis significativamente superiores de sintomas somáticos e psíquicos. Todavia, quando influência tão intensa é observada sobre um instrumento utilizado na prática clínica para direcionamento e acompanhamento terapêutico, certos questionamentos devem ser levantados.

1) A validade do B-K, anteriormente questionada por Alder et al. ${ }^{4}$, parece ter sua fragilidade acrescida pelos resultados encontrados neste estudo. 
2) Apesar da influência da menopausa sobre o humor, discutida pela literatura em estudos conduzidos em indivíduos da comunidade ${ }^{11}$, entre pacientes em atendimento ambulatorial, essa relação parece mais importante quando considerada de forma inversa $^{5,12}$. Por esse motivo, a utilização do B-K em ambiente clínico precisa ser conjugada com a correta detecção dos transtornos mentais.

3) Sem isso, pacientes com transtornos depressivos ou ansiosos moderados a graves serão submetidas a tratamentos de reposição hormonal, para os quais se mostrarão pouco eficazes ${ }^{13}$, retardando o uso de antidepressivos ou ansiolíticos.

Naturalmente, a melancolia foi o sintoma mais específico da presença de algum transtorno depressivo ou ansioso, mostrando-se 6,8 vezes mais prevalente entre as paciente do GTDA. Outros sintomas habitualmente encontrados nos manuais diagnósticos para caracterização dos transtornos depressivos ou ansiosos mostraram-se mais intensos, como a insônia, ou mais prevalentes, como a fraqueza, no GTDA. Observamos ainda uma tendência de queixas mais intensas e prevalentes de fogachos no GTDA, dado comumente encontrado com maior significância em outros estudos ${ }^{14,15}$ e que provavelmente não se mostrou estatisticamente significativo em virtude da pequena amostra avaliada.

A dormência das extremidades (parestesia) foi o sintoma somático significativamente mais referido e mais intenso entre as pacientes do GTDA. Foi o segundo sintoma mais referido por esse grupo enquanto que, no GC, ficou em sétimo lugar. Esse dado evidencia a capacidade dos transtornos depressivos e ansiosos de amplificarem também queixas somáticas. A intensificação particular da parestesia merece especial atenção. Isso porque sintomas somáticos freqüentes nos transtornos depressivos ou ansiosos, como palpitação e vertigem $^{16,17}$, não se mostraram significativamente mais prevalentes no GTDA, e outro sintoma freqüente, a cefaléia ${ }^{16,17}$, mostrou-se ainda menos prevalente e menos intenso no GTDA, apesar de ambos os dados não terem sido estatisticamente significativos. Esse destaque da parestesia parece ser uma particularidade da amostra estudada, podendo ser influenciado por fatores como a cultura, a condição socioeconômica ${ }^{18}$, a concentração de transtornos depressivos e ansiosos de predominante sintomatologia somática em centros de atendimento primário e a forma como 0 atendimento é prestado ${ }^{19}$.

Apesar da limitação da pequena amostra, pudemos observar o significativo viés dos transtornos depressivos e ansiosos sobre as pacientes em tratamento por queixas referentes à menopausa. Por esse motivo, o perfil sintomático de cada paciente deve ser sempre observado, evitando o ginecologista basear-se na intensidade global dos sintomas para decidir sobre o tratamento. Acreditamos que o presente estudo foi capaz de contribuir para o apontamento de sintomas mais relacionados aos transtornos mentais entre mulheres brasileiras em acompanhamento ambulatorial para menopausa.

\section{Referências}

1. Stewart DE, Boydell KM. Psychologic distress during menopause: associations across the reproductive life cycle. Int J Psychiatry Med. 1993;23:157-62.

2. Hay AG, Bancroft J, Johnstone EC. Affective symptoms in women attending a menopause clinic. Br J Psychiatry. 1994;164:5136.

3. Kupperman HS, Wetchler BB, Blatt MH. Contemporary therapy of the menopausal syndrome. JAMA. 1959;171:1627-37.

4. Alder E. The Blatt-Kupperman menopausal index: a critique. s. 1998;29:19-24.

5. Pedro AO, Pinto-Neto AM, Costa-Paiva L, Osis MJ, Hardy E. Procura de serviço médico por mulheres climatéricas brasileiras, Brasil. Rev Saude Publica. 2002;36(4):484-90.

6. Whitehead M. The Pieter Van Keep memorial lecture. In: Berg G, Hammar M, editors. The modern management of the menopause: a perspective for the 21st century. New York: Parthenon; 1994. p. 1-13.

7. Veras AB, Rassi A, Valença AM, Nardi AE. Prevalência de transtornos depressivos e ansiosos em uma amostra ambulatorial brasileira de mulheres na menopausa. Rev Psiquiatr RS. 2006;28:130-4.

8. Sheehan DV, Lecrubier Y, Janavs J, Knapp E, Weiller E, Sheehan $\mathrm{M}$, et al. Mini International Neuropsychiatric Interview Version 4.4 (MINI). Tampa: University of South Florida; 1996.

9. de Waal MW, Arnold IA, Spinhoven P, Eekhof JA, van Hemert AM. The reporting of specific physical symptoms for mental distress in general practice. J Psychosom Res. 2005;59:89-95.

10. Callegari C, Buttarelli M, Cromi A, Diurni M, Salvaggio F, Bolis P. Female psychopathologic profile during menopausal transition: A preliminary study. Maturitas. 2007;56:447-51.

11. Maartens LW, Knottnerus JA, Pop VJ. Menopausal transition and increased depressive symptomatology: a community based prospective study. Maturitas. 2002;42:195-200.

12. Wojnar M, Drozdz W, Araszkiewicz A, Szymanski W, NawackaPawlaczyk D, Urbanski R. [Prevalence of depressive disorders among perimenopausal women seeking gynecological services]. Psychiatr Pol. 2003;37:811-24.

13. Soares CN, Arsenio H, Joffe H, Bankier B, Cassano P, Petrillo LF, et al. Escitalopram versus ethinyl estradiol and norethindrone acetate for symptomatic peri- and postmenopausal women: impact on depression, vasomotor symptoms, sleep, and quality of life. Menopause. 2006;13:7806.

14. Freeman EW, Sammel MD, Lin H, Gracia CR, Kapoor S, Ferdousi $\mathrm{T}$. The role of anxiety and hormonal changes in menopausal hot flashes. Menopause. 2005;12:258-66.

15. Juang KD, Wang SJ, Lu SR, Lee SJ, Fuh JL. Hot flashes are associated with psychological symptoms of anxiety and depression in peri- and post- but not premenopausal women. Maturitas. 2005;52:119-26.

16. Hamilton, M. The assessment of anxiety states by rating. Br J Med Psychol. 1959;32:50-5.

17. Hamilton, M. A rating scale for depression. J Neurol Neurosurg Psychiatry. 1960;23:56-62. 
18. Thomas, JL; Brantley, PJ. Factor structure of the center for epidemiologic studies depression scale in low-income women attending primary care clinics. Eur J Psychol Assess. 2004;20(2):106-15.
19. Simon GE, VonKorff M, Piccinelli M, Fullerton C, Ormel J. An international study of the relation between somatic symptoms and depression. N Engl J Med. 1999;341:1329-35. 